科 学 通 报

\title{
可积族零曲率表示的统一结构
}

\author{
马 文 秀 \\ (但旦大学数学呼究斦, 上海 200433)
}

\section{关键词可积系绕、等橧与非等谱问题、娄曲晕表示、特征算子方程、Lax 算子}

设 $x, t \in \mathbb{R}, u=\left(u_{1}, \cdots, u_{q}\right)^{T}, u_{i}=u_{i}(x, t), 1 \leqslant i \leqslant q$, 令 $\mathscr{B}$ 表示 $C^{\infty}$ 可微函数 $P(x$, $t, u)$ 的全体, $\mathscr{B}^{r}=\left\{\left(P_{1}, \cdots, P_{r}\right)^{\top} \mid P_{1} \in \mathscr{B}\right\}, \mathscr{V}^{r}$ 表示 $C^{\infty}$ 可微线性算子 $\Phi=\Phi(x, t, u)$; $\mathscr{B}^{r} \rightarrow \mathscr{B}^{r}$ 的全体 ${ }^{[1]}$, 用 $\mathscr{W}(r)$ 表示所有矩阵 乘 积算子 $w=w(u, \lambda)=\left(w_{i j}\right)_{r \times r}$, 这 琹 $w_{i j}=w_{i j}(u, \lambda)$ 关于作为 $x$ 的函数 $u$ 是 $C^{\infty}$-Gateaux 可微且关于谱参数 $\lambda$ 是 $C^{\infty}$ 可微的函 数. 设 $K, s \in \mathscr{B} q, w \in \mathscr{W}(r)$, 定义 Gateaux 导数为

$$
K^{\prime}[s]=-\left.\frac{\partial}{\partial \varepsilon}\right|_{\varepsilon=0} K(u+\varepsilon s), \quad w^{\prime}[s]=\left.\frac{\partial}{\partial \varepsilon}\right|_{c=0} w(u+\varepsilon s),
$$

$\mathscr{B}^{9}$ 关于运算 $[K, s]=K^{\prime}[s]-s^{\prime}[K], K, s \in \mathscr{B}^{q}$ 是一个 Lie 代数 ${ }^{[2]}$.

考虑谱问题

$$
\left\{\begin{array}{l}
\varphi_{x}=U \varphi=U(u, \lambda) \varphi, \\
\varphi_{t}=V \varphi=V(u, \lambda) \varphi,
\end{array} U, V \in \mathscr{W}(r),\right.
$$

它的可积性条件即为零曲率方程

$$
U_{t}-V_{x}+[U, V]=0 .
$$

定义 1 如果-一个发展方程

$$
u_{s}=K=K(x, t, u), K \in \mathscr{B}^{q}
$$

等价于零曲率方程 (2), 就称(3)具有零曲率表示(2), $V$ 称为(3)相应的一个 Lax 算子.

零曲率方程 (2)不可积系统理论中起着重要作用, 一般地可积系统都是在方程 (2)的基础 上生成的,并利用谱问题(1)可产生可积系的无穷多守恒量 ${ }^{[3-5]}$.

本文对可积族(他括等谱 $\left(\lambda_{t}=0\right)$ 和非等谱 $\left(\lambda_{t}=\lambda^{n}, n \geqslant k\right)$ 情形)提出了零曲率表示 的统一结构, 其基本焽想是把可积族的递推性转化为一个特征算子方程. 继而应用这个框架, 对耦合 $K d V$ 可积族给出了相应的特征算子方程的一个解及初始非等谱向量场, 进一步生成 了等谱与非等谱耦合 $K d V$ 可积族的二族 $\mathrm{Lax}$ 算子.

下面总假定 $U \in \mathscr{W}(r)$ 的 Gateaux 导数算子 $U^{\prime}$ 是单的. 注意 $U_{t}=U^{\prime}\left[u_{\imath}\right]+\lambda_{s} U_{\lambda}$, 我们可知

命题 1 发展方程(3)具有零曲率衣示(2)当且仅当

$$
U^{\prime}[K]+\lambda_{x} U_{\lambda}-V_{x}+[U, V]=0 .
$$

假设我们有等谱的可积族

$$
u_{t}=K_{m}=\Phi^{m} f_{0}, \Phi \in \mathscr{V}^{q}, f_{0} \in \mathscr{B}^{q}, m \geqslant 0,
$$

1992-03-27 收稿. 
一般地这里的算子 $\Phi$ 是由 $\varphi_{x}=U \varphi$ 确定的遗传对称 ${ }^{[6]}$. 对任意给定的 $X \in \mathscr{B}^{9}$, 构造一个 关于算子 $\Omega \in \mathscr{W}(\boldsymbol{r})$ 的特征算子方程

$$
U^{\prime}[\Phi X]-\lambda U^{\prime}[X]=[\Omega, U]+\Omega_{x},
$$

底下总假定 (6)有解, 记一解为 $\Omega=Q(X)$.

定理 1 设算子 $A_{0} \in \mathscr{W}(r)$ 满足 $U^{\prime}\left[f_{0}\right]-A_{0 x}+\left[U, A_{0}\right]=0$, 并令

$$
V_{m}=\sum_{i=0}^{m} \lambda^{m-i} A_{i}=\lambda^{m} A_{0}+\sum_{i=1}^{m} \lambda^{m-i} \Omega\left(K_{i-1}\right), \quad m \geqslant 0,
$$

则我们有

$$
U^{\prime}\left[K_{m}\right]-V_{m x}+\left[U, V_{m}\right]=0, m \geqslant 0,
$$

因此对每一 $m \geqslant 0$, 可积系 $u_{t}=K_{m}$ 具有等谱 $\left(\lambda_{t}=0\right)$ 的零曲率 表 示 $U_{t}-V_{m x}+[U$, $\left.V_{m}\right]=0$.

证 设 $m \geqslant 0$, 我们可计算如下:

$$
\begin{aligned}
{\left[U, V_{m}\right] } & =\left[U, \sum_{i=0}^{m} \lambda^{m-i} A_{i}\right]=\sum_{i=0}^{m} \lambda^{m-i}\left[U, A_{i}\right] \\
& =\lambda^{m}\left[U, A_{0}\right]+\sum_{i=1}^{m} \lambda^{m-i}\left[U, Q\left(K_{i-1}\right)\right] \\
& =\lambda^{m}\left(A_{0 x}-U^{\prime}\left[K_{0}\right]\right)+\sum_{i=1}^{m} \lambda^{m-i}\left(Q\left(K_{i-1}\right)_{x}-U^{\prime}\left[K_{i}\right]-\lambda U^{\prime}\left[K_{i-1}\right]\right) \\
& =\lambda^{m} A_{0 x}+\sum_{i=1}^{m} \lambda^{m-i} Q\left(K_{i-1}\right)_{x}-U^{\prime}\left[K_{m}\right] \\
& =V_{m x}-U^{\prime}\left[K_{m}\right],
\end{aligned}
$$

此即为 $(8)$ 式. 余由命题 1 知成立. 证毕.

再假设我们有非等谱的可积族

$$
u_{t}=\sigma_{n}=\Phi^{n} g_{0}, g_{0} \in \mathscr{B B}^{q}, n \geqslant 0,
$$

这里算子 $\Phi$ 就是等谱族 (5)中的算子 $\Phi$.

定理 2 设 $k \in \mathbf{Z}_{0}^{+}$和算子 $B_{0} \in \mathscr{W}(r)$ 满 足 $U^{\prime}\left[g_{0}\right]+\lambda^{k} U_{\lambda}-B_{0 x}+\left[U, B_{0}\right]=0$, 并令

则我们有

$$
w_{n}=\sum_{i=0}^{n} \lambda^{n-i} B_{i}=\lambda^{n} B_{0}+\sum_{i=1}^{n} \lambda^{n-i} \Omega\left(\sigma_{i-1}\right), n \geqslant 0,
$$

$$
U^{\prime}\left[\sigma_{n}\right]+\lambda^{n+k} U_{\lambda}-w_{n x}+\left[U, w_{n}\right]=0, n \geqslant 0,
$$

因此对每一 $n \geqslant 0$, 可积系 $u_{t}=\sigma_{n}$ 具有非等谱 $\left(\lambda_{t}=\lambda^{n+k}\right)$ 的零曲率表示 $U_{t}-w_{n x}+$ $\left[U, w_{n}\right]=0$.

证 设 $n \geqslant 0$. 注意假设与特征算子方程(6)我们有

$$
\begin{aligned}
{\left[U, w_{n}\right] } & =\left[U, \sum_{i=0}^{n} \lambda^{n-i} B_{i}\right]=\sum_{i=0}^{n} \lambda^{n+i}\left[U, B_{i}\right] \\
& =\lambda^{n}\left[U, B_{0}\right]+\sum_{i=1}^{n} \lambda^{n-i}\left[U, \Omega\left(\sigma_{i-1}\right)\right]
\end{aligned}
$$




$$
\begin{aligned}
& =\lambda^{n}\left(B_{0 x}-U^{\prime}\left[\sigma_{0}\right]-\lambda^{k} U_{\lambda}\right)+\sum_{i=1}^{n} \lambda^{n-i}\left(Q\left(\sigma_{i-1}\right)_{x}-U^{\prime}\left[\sigma_{i}\right]-\lambda U^{\prime}\left[\sigma_{i-1}\right]\right) \\
& =\lambda^{n} B_{0 x}-\lambda^{n+k} U_{\lambda}+\sum_{i=1}^{n} \lambda^{n-i} Q\left(\sigma_{i-1}\right)_{x}-U^{\prime}\left[\sigma_{n}\right] \\
& =w_{n x}-\lambda^{n+k} U_{\lambda}-U^{\prime}\left[\sigma_{n}\right],
\end{aligned}
$$

此就是(11)式. 其余结果明显成立. 证毕.

下面开始应用上述统一结构来考虑耦合 $K d V$ 可积族 ${ }^{[7,8]}$

其中

$$
\begin{array}{r}
u_{1}=K_{m}=\Phi^{m} f_{0}=\Phi^{m} u_{x} . \\
u=\left[\begin{array}{c}
v_{0} \\
v_{1} \\
\vdots \\
v_{q-1}
\end{array}\right], \quad \Phi=\left[\begin{array}{cc|c} 
& 0 & R_{0}^{*} \\
\hline 1 & 0 & R_{1}^{*} \\
& \ddots & \vdots \\
0 & 1 & R_{q-1}^{*}
\end{array}\right],
\end{array}
$$

而 $R_{i}^{*}=\left(v_{i}-\frac{1}{2} I v_{i x}+\frac{1}{4} \delta_{i l} \partial^{2}\right)^{*}=v_{i}+\frac{1}{2} v_{i x} I+\frac{1}{4} \delta_{i l} \partial^{2}, 0 \leqslant i \leqslant q-1, I=\partial^{-1}$. 它相应于谱问题 $[7,8]$

$$
\psi_{x x}+Q \psi=0, Q=Q(u, \lambda)=\lambda^{-l} \sum_{i=0}^{q} \nu_{i} \lambda^{i}, v_{q}=-1, q \geqslant 1,0 \leqslant l \leqslant q-1 .
$$

令 $\varphi_{1}=\psi, \varphi_{2}=\psi_{x}$, 则(13)式可改写为

$$
\varphi_{s}=U \varphi, \quad \varphi=\left[\begin{array}{c}
\varphi_{1} \\
\varphi_{2}
\end{array}\right], \quad U=\left[\begin{array}{rr}
0 & 1 \\
-Q & 0
\end{array}\right] \text {. }
$$

社显地

$$
U^{\prime}[X]=\left[\begin{array}{cc}
0 & 0 \\
-\lambda^{-l} \sum_{i=1}^{q} X_{i} \lambda^{i-1} & 0
\end{array}\right], X=\left(X_{1}, \cdots, X_{q}\right)^{T} \in \mathscr{B} q,
$$

因此 $U^{\prime}$ 是单射. 下面我们来求相应的特征算子方程的算子特解. 设 $X=\left(X_{1}, \cdots, X_{q}\right)^{T} \in$ $\mathscr{B}^{q}, \Phi X=\left((\Phi X)_{1}, \cdots,(\Phi X)_{q}\right)^{T}, Q=\left[\begin{array}{ll}Q^{(1)} & Q^{(2)} \\ Q^{(3)} & -Q^{(1)}\end{array}\right] \in \mathscr{W}(2)$, 则

$$
\begin{aligned}
U^{\prime}[\Phi X]-\lambda U^{\prime}[X] & =-\lambda^{-l}\left[\begin{array}{cc}
0 & 0 \\
(\Phi X)_{1}+\sum_{i=1}^{q-1}\left[(\Phi X)_{i+1}-X_{i}\right] \lambda^{i}-X_{q} \lambda^{q} & 0
\end{array}\right] \\
& =-\lambda^{-l}\left[\begin{array}{cr}
0 & 0 \\
\sum_{i=0}^{q-1}\left(R_{i}^{*} X_{q}\right) \lambda^{i}-X_{q} \lambda^{q} & 0
\end{array}\right], \\
{[\Omega, U]+\Omega_{x} } & =\left[\begin{array}{cc}
-Q Q^{(2)}-\Omega^{(3)}+\Omega_{x}^{(1)} & 2 \Omega^{(1)}+\Omega_{x}^{(2)} \\
2 Q Q^{(1)}+\Omega_{x}^{(3)} & \Omega^{(3)}+Q Q^{(2)}-\Omega_{x}^{(1)}
\end{array}\right] .
\end{aligned}
$$

于是

$$
\left\{\begin{array}{l}
-Q Q^{(2)}-Q^{(3)}+Q_{x}^{(1)}=0,2 Q^{(1)}+Q_{x}^{(2)}=0, \\
2 Q Q^{(1)}+Q_{x}^{(3)}=-\lambda^{-l}\left[\sum_{i=0}^{q-1}\left(R_{i}^{*} X_{q}\right) \lambda^{i}-X_{q} \lambda^{q}\right],
\end{array}\right.
$$

进一步

$$
2 Q Q^{(1)}+Q_{x}^{(3)}=-2\left(\frac{1}{2} Q_{x}+Q \partial+\frac{1}{4} \partial^{3}\right) Q^{(2)}
$$




$$
=-2 \lambda^{-t}\left(\sum_{i=0}^{a-1} \lambda^{i} R_{i}^{*} \partial-\lambda^{i} \partial\right) Q^{(2)}
$$

故可取 $Q^{(2)}=\frac{1}{2} I X_{q}$. 这样我们得到(6)式的一个解:

$$
\boldsymbol{Q}=\boldsymbol{Q}(X)=\left[\begin{array}{cc}
\Omega^{(1)} & Q^{(3)} \\
\Omega^{(3)} & -Q^{(1)}
\end{array}\right]=\left[\begin{array}{cc}
-\frac{1}{4} X_{q} & \frac{1}{2} I X_{q} \\
-\frac{1}{2} Q I X_{q}-\frac{1}{4} X_{q x} & \frac{1}{4} X_{q}
\end{array}\right], X=\left[\begin{array}{c}
X_{1} \\
\vdots \\
X_{q}
\end{array}\right] \in \mathscr{B} q
$$

又可取 $A_{0}=U$, 于是按定理 1 我们就可得到等谱可积族 (12)的一种零曲率表示. 特别地可 积系

$$
u_{1}=K_{1}=\Phi f_{0}=\left[\begin{array}{c}
v_{0} v_{q-1, x}+\frac{1}{2} v_{0 x} v_{q-1}+\frac{1}{4} \delta_{0 l} v_{q-1, x x x} \\
v_{0 x}+v_{1} v_{q-1, x}+\frac{1}{2} v_{1 x} v_{q-1}+\frac{1}{4} \delta_{1 l} v_{q-1, s x x} \\
\vdots \\
v_{q-1, x}+v_{q-2} v_{q-1, x}+\frac{1}{2} v_{q-2, x} v_{q-1}+\frac{1}{4} \delta_{q-2, l} v_{q-1, x x x} \\
v_{q-2, x}+\frac{3}{2} v_{q-1} v_{q-1, x}+\frac{1}{4} \delta_{q-1, l} v_{q-1, x \leq x}
\end{array}\right]
$$

有等谱 $\left(\lambda_{1}=0\right)$ 的 $\operatorname{Lax}$ 算子

$$
\begin{aligned}
V_{1} & =\lambda A_{0}+A_{1}=\lambda A_{0}+\Omega\left(K_{0}\right) \\
& =\left[\begin{array}{cc}
-\frac{1}{4} v_{q-1, x} & \lambda+\frac{1}{2} v_{q-1} \\
-\left(\lambda+\frac{1}{2} v_{q-1}\right) \theta-\frac{1}{4} v_{q-1, x} & \frac{1}{4} v_{q-1, x}
\end{array}\right] .
\end{aligned}
$$

再考虑非等谱族情形. 求解关于 $g_{0} \in \mathscr{B}^{q}$ 和 $B_{0} \in \mathscr{W}(2)$ 的方程

$$
U^{\prime}\left[g_{0}\right]+\lambda^{k} U_{\lambda}-B_{0 x}+\left[U, B_{0}\right]=0 \text {. }
$$

取 $k=1, B_{0}=\left(\begin{array}{rr}B_{0}^{(1)} & B_{0}^{(2)} \\ B_{0}^{(3)} & -B_{0}^{(1)}\end{array}\right) \in \mathscr{P}(2), B_{0}^{(2)} \in \mathscr{B}$, 则可得

$$
\begin{aligned}
g_{0}= & \left(q v_{0}+\frac{1}{2}(q-l) x v_{0 x},(q-1) v_{1}+\frac{1}{2}(q-l) x v_{l x}, \cdots, v_{q-1}\right. \\
& \left.+\frac{1}{2}(q-l) x v_{q-1, x}\right)^{T} \\
B_{0}= & {\left[\begin{array}{cc}
-\frac{1}{4}(q-l) & -\frac{1}{2}(q-l) x \\
-\frac{1}{2}(q-l) x Q & \frac{1}{4}(q-l)
\end{array}\right] }
\end{aligned}
$$

这样我们得到非等谱族

$$
u_{i}=\sigma_{n}=\Phi^{n} g_{0}, n \geqslant 0,
$$

这里的推导过程表明定理 2 实际上也给出了一个寻求非等谱族的方法. 按定理 2 对每一 $n \geqslant$ 0 , 可积系 $u_{t}=\sigma_{n}$ 有非等谱 $\left(\lambda_{s}-\lambda^{n+1}\right)$ 的零曲率表示. 我们指出当 $l=0$ 时,在(18)中可 
取 $k=0, B_{0}=0$, 此时(18)式有解

$$
g_{0}=\left(-v_{1},-2 v_{2}, \cdots,-(q-1) v_{q-1}, q\right)^{T} .
$$

对等谱族 (12), 重 Hamilton 结构、无穷多守恒律及相容的 Poisson 括弧等都已被 提 出 $^{[7-10]}$. 我们还想说明一下定理 1,2 中的条件均是自然的, 因为即要求 $u_{t}=\dot{K}_{0}$ 和 $u_{t}=\sigma_{0}$ 具 有等谱 $\left(\lambda_{t}-0\right)$ 和非等谱 $\left(\lambda_{t}-\lambda^{k}\right)$ 的零曲率表示. 这里得到的二族 $\operatorname{Lax}$ 算子(7), (10) 式的 Lie 代数结构, 我们将另文讨论. 然而特征算子方程 (6)的几何内涵如何和遗传对称算 子 $\Phi$ 与算子解 $Q$ 之间内蕴关系如何尚需进一步探讨.

致谢 作者衰心感谢谷超豪教授和胡和生教授的热情支持及指导.

\section{参孝文献}

[1] 马文秀,科学通报, 1992, 37 (1): 8-11.

[2] Bowman, S., Math. Proc. Camb. Phil. Soc., 1987, 102: 173-189.

[ 3 ] Newell, A. C., Solitons in Mathematics and Physics, SIAM, 1985.

[ 4 ] Tu, G. Z., J. Phy: A: Mazh. Gen., 1989, 22: 2375-2392.

[ 5 ] Alberty, T. M., Koikawa, T., Sasaki, R., Physica D, 1982, 5: 43-65.

[ 6 ] Fokas, A. S., Anderson, R. L., J. Math. Phys., 1982, 23: 1066-1073.

[7] Boiti, M., Caudrey, P. J., Pempinelli, F., Il Nuovo Cimento B, 1984, 83: 71-87.

[8] 马文秀,非线性演化方程的广义 Hamilton 结构之研究,硕士论文,中国科学院计算中心, 1985.

[ 9 ] Antonowicz, M., Fordy, A. P., in Nonlinear Evolutions (ed. Leon, J. J. P.), World Scientific, 1988, 145-159.

[10] Fordy, A. P., Reyman, A. G., Semenov-Tian-Shansky, M. A., Lett. Math. Phys, 1989, 17: $25-29$. 